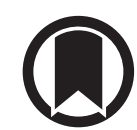

CrossMark

\title{
Neutrophil extracellular traps in ex vivo lung perfusion perfusate predict the clinical outcome of lung transplant recipients
}

\author{
To the Editor:
}

Lung transplantation is lifesaving for patients with end-stage lung diseases. However, a scarcity of donor lungs suitable for transplantation limits the number of procedures that can be performed. Furthermore, major obstacles to successful lung transplantation still exist; primary graft dysfunction (PGD), the leading cause of early morbidity and mortality post-lung transplant, develops in $\sim 15-30 \%$ of recipients [1]. Normothermic ex vivo lung perfusion (EVLP) maintains donor lungs in a physiological state through perfusion and ventilation, so that the lungs can be assessed, treated and accepted for transplantation, thereby increasing the donor pool and alleviating the severe shortage of viable donor lungs [2].

In addition to donor lung assessment, EVLP provides a unique platform to study cellular processes occurring in donor lungs. EVLP perfusate is a unique, noninvasive source of biological sampling, which offers valuable information about the state of the donor lung. Rapid, molecular diagnostic testing could be an important addition to assessment of donor lungs, and may help improve outcome [3-7]. Detection of injurious markers in EVLP perfusate may also help inform future targeted treatments for the lungs, which could be administered directly to the organ through the EVLP circuit.

In order to develop a reliable molecular assessment of donor lungs, a stable, easily quantifiable biomarker that reflects donor lung injury is required. Neutrophil extracellular traps (NETs) are structures comprised of extracellular neutrophil DNA in a complex with antimicrobial granular proteins, such as neutrophil elastase (NE) and myeloperoxidase. Neutrophils form NETs in response to innate immune challenges. Although NETs are generally considered antibacterial, several recent studies suggest that the cytotoxicity of NETs can be highly detrimental to lung and other tissue through complement activation, exposed histone cytotoxicity, promotion of thrombosis, and propagation of neutrophil recruitment and activation [8-11]. NETs have been specifically implicated in a variety of lung disease and injury states, including ventilator-induced lung injury and PGD $[12,13]$.

NETs play an important role in lung injury, but their role in lung transplantation is largely unknown. As NETs are a quantifiable measure of the innate immune response to a variety of inflammatory stimuli, this study aimed to determine whether NETs are detectable in EVLP perfusate, indicating their production by neutrophils in donor lungs. Furthermore, this study assessed the ability of NETs to serve as a relevant biomarker of donor lung injury. We report that elevated NET levels in donor lung EVLP perfusate are associated with worse recipient outcomes.

Written, informed consent was obtained, and this study was approved by the University Health Network Research Ethics Board. Clinical perfusate samples from consecutive EVLP cases that were performed at our centre between 2009 and 2017, excluding cases that resulted in single lung transplantation $(n=200)$, were taken at the 4th hour of perfusion and snap frozen. Complexes of NE bound to DNA, representative of NETs, were measured using an ELISA. Within the lungs that were transplanted, optical densities were analysed against recipient outcomes in patients who were not in the intensive care unit (ICU) prior to

@ERSpublications

Neutrophil extracellular traps (NETs) are detectable in donor ex vivo lung perfusate, and higher levels of NETs in perfusate are associated with worse recipient outcomes after transplant http://ow.ly/ r4nM30nvZsK

Cite this article as: Caldarone L, Mariscal A, Sage A, et al. Neutrophil extracellular traps in ex vivo lung perfusion perfusate predict the clinical outcome of lung transplant recipients. Eur Respir J 2019; 53: 1801736 [https://doi.org/10.1183/13993003.01736-2018]. 
transplantation (arterial oxygen tension/inspiratory oxygen fraction ratio at ICU arrival, PGD at $72 \mathrm{~h}$, days on the ventilator, and days in the ICU). From the 200 perfusate samples included, 111 were from donation after brain death (DBD) and 89 were from donation after circulatory death (DCD) lungs. To minimise possible confounding factors related to recipient severity of illness, outcomes were only analysed for recipients that were not admitted to the ICU prior to transplantation, resulting in $63 \mathrm{DBD}$ recipients and 52 DCD recipients suitable for analysis. NETs were analysed against recipient outcomes (days on ventilator and days in ICU) using a threshold of 4 days, a clinically relevant cut-off.

The biological processes that occur during brain death differ from those that unfold during cardiac death, and manifest in different potential insults to the lungs [14, 15]. Additionally, females tend to exhibit a more robust innate immune response than males [16], and have been shown to demonstrate higher leukocyte counts compared with males in a rat model of brain death [17]. To account for these biological differences, NETs were analysed in the overall study cohort as well as by sex and donor type. A Bonferroni-Dunn correction was applied to these analyses.

NETs were significantly higher in donor lung perfusate for patients who were on the ventilator for $>4$ days ( $p=0.0422)$, particularly for patients who received female donor lungs $(p=0.0383$; figure $1 \mathrm{a})$. Analyses of donor lung types indicated that the difference was attributable to DCD lungs $(p=0.0160$; figure $1 b$ ) obtained from female donors ( $p=0.0088$; figure $1 b$ ). Length of ICU stay is related to the recipient's days on ventilator. Consistent with this point, results of analysis of perfusate NETs and recipient length of ICU stay were similar to those of recipient days on the ventilator (data not shown).

Regression analyses further show that increased levels of NETs in perfusate were significantly associated with more recipient days on the ventilator $\left(R^{2}=0.1101, p=0.0027\right.$; figure $\left.1 c\right)$. The association between higher NETs and more recipient days on the ventilator was apparent in perfusate from $D C D\left(R^{2}=0.2472\right.$, $\mathrm{p}=0.0018$; figure 1d) but not in DBD lungs $\left(\mathrm{R}^{2}=0.01902, \mathrm{p}=1.000\right)$. When stratified into male and female donors, regardless of the type of donor lung (DCD or DBD), NETs were significantly associated with recipient days on the ventilator in female donor lungs $\left(\mathrm{R}^{2}=0.2306, \mathrm{p}=0.0099\right.$; figure $\left.1 \mathrm{c}\right)$. When stratified into both donor sex and type categories, DCD females demonstrated a very strong association between NETs in the donor perfusate and recipient days on the ventilator $\left(R^{2}=0.5361, p=0.0009\right.$; figure $\left.1 d\right)$. A similar relationship existed even after excluding a patient who received the DCD lung with the highest level of NETs and stayed on the ventilator for the longest time $\left(R^{2}=0.2347, p=0.0206\right.$; figure $\left.1 d\right)$.

This study demonstrates, for the first time, that NETs are detectable in clinical EVLP perfusate. Although NETs have been implicated in lung transplant-related injury [12], no studies to date have examined the role of NETs in donor lungs. The presence of NETs in donor lungs is important as it suggests that NETs form in response to donor lung injury, and are released from the lungs into the circulating perfusate. As the perfusion system mimics physiological circulation, it is plausible that NETs formed in donor lungs could be released into the recipient's circulation upon reperfusion, contributing to post-transplant injury.

Elevated perfusate NETs were significantly associated with increased recipient days on the ventilator. Furthermore, recipients who received lungs with significantly higher levels of NETs in the donor perfusate were on the ventilator for $>4$ days. NETs are appealing as a potential biomarker of lung injury as they represent innate immune activation from a wide variety of inciting stimuli [18], suggesting that they could be formed in response to a spectrum of different clinical injuries. Furthermore, NETs are relatively stable [19] and detectable with ELISA, a common and inexpensive laboratory technique. Although standard EVLP practice in our centre is $4-6 \mathrm{~h}$ of perfusion, our group and others have shown that injured lungs can be successfully perfused for $\leqslant 12 \mathrm{~h}$ in a large animal model [20-22]. Therefore, it is plausible that detection of NETs could occur in a clinically relevant timeframe to inform decision-making.

The positive correlation between perfusate NETs and recipient days on the ventilator or the length of stay in ICU was detectable in DCD but not in DBD lung perfusates. One possible reason for the difference between these groups is the biology of the lung injury that occurs during brain death and the associated catecholamine storm, which overwhelms the circulation with pro-inflammatory cytokines [14]. The overload of inflammatory mediators could result in NET production from brain-death related cytokines as well as donor lung injury. Interestingly, in another study of biomarkers in EVLP perfusate, we also found significant associations only in the DCD cohort [4]. Therefore, it is possible that the ex vivo perfusion incites a process of metabolic reawakening that unfolds differently in DCD and DBD lungs. The differences observed between the two donor groups is important to consider for development of future biomarkers, as this suggests that different biological factors influence detectable proteins in donor lungs. The underlying mechanisms merit further investigation.

In addition to differences by donor type, an association between perfusate NETs and recipient days on the ventilator was observed in female donor lungs compared with male donor lungs. Sex differences have been 

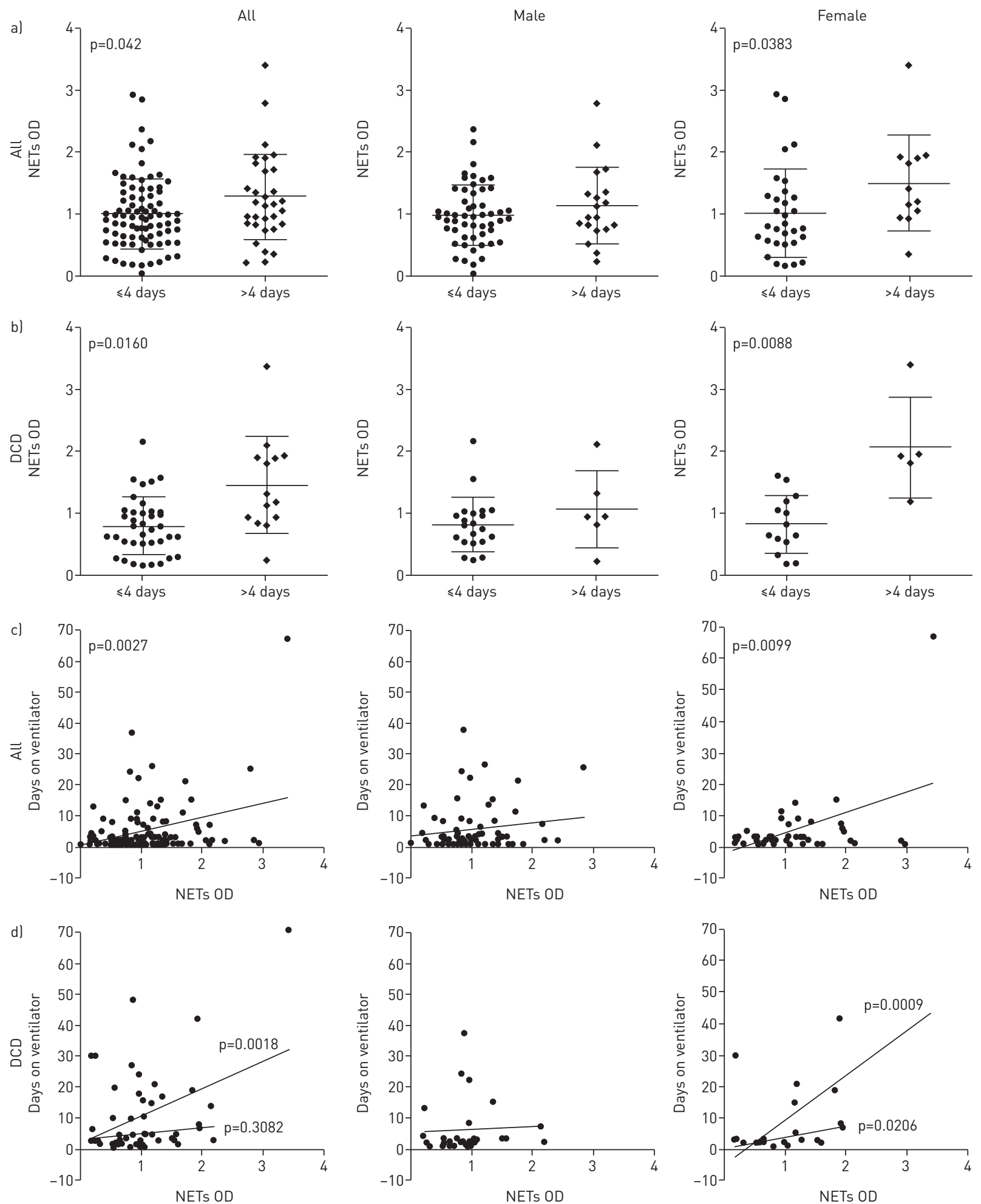

FIGURE 1 Clinical ex vivo lung perfusion (EVLP) perfusate was sampled at $4 \mathrm{~h}$ of perfusion. Neutrophil extracellular traps (NETs) were measured in these samples using neutrophil elastase bound to DNA (NE-DNA) sandwich NET ELISA (optical density (OD)). a) NET concentrations were higher in donor lung perfusate for recipients who were on the ventilator for $>4$ days $(p=0.042)$, particularly in female donor lung perfusate for recipients on the ventilator for $>4$ days $(p=0.0383)$. b) NET concentrations were higher in donation after circulatory death (DCD) lung perfusate for recipients on the ventilator for $>4$ days ( $p=0.0160$ ), particularly in DCD female donor lung perfusate ( $p=0.0088)$. $c)$ NETs in donor lung perfusate were positively correlated with recipient days on the ventilator in the overall study cohort $\left(R^{2}=0.1101, p=0.0027\right)$ as well as in female donor lung perfusate $\left(R^{2}=0.2306, p=0.0099\right)$. d) NET concentration in $D C D$ lung perfusate was positively correlated with recipient days on the ventilator $\left(R^{2}=0.2472, p=0.0018\right)$, and this correlation was apparent between $D C D$ female lung perfusate and recipient days on the ventilator $\left(R^{2}=0.5361\right.$, $p=0.0009$ ). Even after excluding one recipient, who had the highest NET concentration and the highest ventilator days, the NET concentration in the female DCD lung perfusate was positively correlated with recipient days on the ventilator $\left(R^{2}=0.2347, p=0.0206\right)$. 
described with regard to NETosis [23] as well as lung disease [24], but the potential effects of sex differences on NETs in lung transplantation have not been described. Our observation that NETs originating from female donors are more strongly associated with recipient outcomes suggests that NETs may form in female lungs in a manner more clearly dependent on the severity of donor lung injury. Although further investigation is needed, it is possible that this observation is related to the more robust innate immune response seen in females. This response can contribute to worse clinical outcomes in lung disease (for instance, females are more likely to develop acute respiratory distress syndrome than males [25]) but may explain why NETs are related to recipient outcomes in this circumstance. Overall, this study shows that donor biological characteristics are important to consider in the development of biomarkers for organ transplantation.

We report here the first description of NETs in clinical EVLP perfusate, indicating that NETs form in human donor lungs are released into circulating perfusate. Furthermore, we show that elevated NETs in donor perfusate are associated with more recipient days on the ventilator, and that this association is seen in DCD female donor lungs. Our results suggest that NETs are a novel, reliable and easy to measure biomarker to predict recipient outcomes after lung transplantation.

Lindsay Caldarone ${ }^{1,2}$, Andrea Mariscal ${ }^{1,2,3}$, Andrew Sage ${ }^{1}$, Meraj Khan $^{4}$, Stephen Juvet ${ }^{1,2,3}$, Tereza Martinu ${ }^{1,2,3}$, Ricardo Zamel ${ }^{1}$, Marcelo Cypel ${ }^{1,2,3}$, Mingyao Liu $^{1,2,3}$, Nades Palaniyar $^{2,4,5}$ and Shaf Keshavjee ${ }^{1,2,3}$

${ }^{1}$ Latner Thoracic Surgery Research Laboratories, Toronto General Research Institute, Toronto, Canada. ${ }^{2}$ Institute of Medical Science, University of Toronto, Toronto, ON, Canada. ${ }^{3}$ Toronto Lung Transplant Program, University Health Network, Toronto, ON, Canada. ${ }^{4}$ Translational Medicine, Peter Gilgan Centre for Research and Learning, The Hospital for Sick Children, Toronto, ON, Canada. ${ }^{5}$ Laboratory Medicine and Pathobiology, University of Toronto, Toronto, ON, Canada.

Correspondence: Shaf Keshavjee, Toronto Lung Transplant Program, University Health Network, University of Toronto, Toronto, ON, M5G 2C4, Canada. E-mail: shaf.keshavjee@uhn.ca

Received: Sept 112018 | Accepted after revision: Dec 202018

Conflict of interest: L. Caldarone has nothing to disclose. A. Mariscal has nothing to disclose. A. Sage has nothing to disclose. M. Khan reports receiving grants from Cystic Fibrosis Canada (grant number 3180) and from Mitacs, during the conduct of the study. S. Juvet has nothing to disclose. T. Martinu has nothing to disclose. R. Zamel has nothing to disclose. M. Cypel is a founder of Perfusix Canada Inc., which has provided ex vivo lung perfusion (EVLP) services to the University Health Network (Toronto, ON, Canada) in the past. Due to a conflict of interest relating to EVLP activities as a lung transplant surgeon at the institution, M. Cypel did not receive any payments from Perfusix Canada Inc. Furthermore, with respect to the provision of EVLP services, Perfusix Canada Inc. is a non-profit company that does not generate profit from EVLP activities provided for University Health Network patients. M. Cypel is a co-founder of XOR Labs Toronto Inc., a company dedicated to the development of EVLP machines. The XOR EVLP machine is at development phase and was not used in the performance of this study. Lung Bioengineering (LBI) acquired PXCA in 2015, a company that was co-founded by M. Cypel. Currently, M. Cypel is a paid consultant for LBI. Consultants give strategic advice to LBI lung perfusion centre as members of its scientific advisory board. The samples obtained in the current study were collected from consented University Health Network patients with the major aim of developing biomarkers to predict donor lung quality so that safer transplantation can be performed. M. Liu is a co-founder of XOR Labs Toronto Inc., a company dedicated to the development of EVLP machines. The XOR EVLP machine is at development phase and was not used in the performance of this study. N. Palaniyar reports receiving grants from Cystic Fibrosis Canada (grant number 3180) and from the Canadian Institute of Health Research (gran number MOP-2 111012), during the conduct of the study. S. Keshavjee is the co-founder and Chief Scientific Officer of Perfusix Canada Inc. and XOR Labs Toronto Inc. S. Keshavjee has received the following, outside the submitted work: grants from XVIVO Perfusion for research and providing clinical study support for other studies; and personal fees for acting as a consultant for Lung Bioengineering, United Therapeutics.

\section{References}

1 Diamond JM, Arcasoy S, Kennedy CC, et al. Report of the International Society for Heart and Lung Transplantation Working Group on primary lung graft dysfunction, part II: Epidemiology, risk factors, and outcomes - A 2016 Consensus Group statement of the International Society for Heart and Lung Transplantation. J Heart Lung Transplant 2017; 36: 1104-1113.

2 Machuca TN, Mercier O, Collaud S, et al. Lung transplantation with donation after circulatory determination of death donors and the impact of ex vivo lung perfusion. Am J Transplant 2015; 15: 993-1002.

3 Hashimoto $\mathrm{K}$, Cypel M, Kim H, et al. Soluble adhesion molecules during ex vivo lung perfusion are associated with posttransplant primary graft dysfunction. Am J Transplant 2017; 17: 1396-1404.

4 Machuca TN, Cypel M, Zhao Y, et al. The role of the endothelin-1 pathway as a biomarker for donor lung assessment in clinical ex vivo lung perfusion. J Heart Lung Transplant 2015; 34: 849-857.

5 Hsin MK, Zamel R, Cypel M, et al. Metabolic profile of ex vivo lung perfusate yields biomarkers for lung transplant outcomes. Ann Surg 2018; 267: 196-197.

6 Andreasson ASI, Borthwick LA, Gillespie C, et al. The role of interleukin-1beta as a predictive biomarker and potential therapeutic target during clinical ex vivo lung perfusion. J Heart Lung Transplant 2017; 36: 985-995.

7 Machuca TN, Cypel M, Yeung JC, et al. Protein expression profiling predicts graft performance in clinical ex vivo lung perfusion. Ann Surg 2015; 261: 591-597. 
8 Wang $\mathrm{H}$, Wang $\mathrm{C}$, Zhao $\mathrm{MH}$, et al. Neutrophil extracellular traps can activate alternative complement pathways Clin Exp Immunol 2015; 181: 518-527.

9 Saffarzadeh M, Juenemann C, Queisser MA, et al. Neutrophil extracellular traps directly induce epithelial and endothelial cell death: a predominant role of histones. PLoS One 2012; 7: e32366.

10 Fuchs TA, Brill A, Duerschmied D, et al. Extracellular DNA traps promote thrombosis. Proc Natl Acad Sci USA 2010; 107: 15880-15885.

11 Sabbione F, Keitelman IA, Iula L, et al. Neutrophil extracellular traps stimulate proinflammatory responses in human airway epithelial cells. J Innate Immun 2017; 9: 387-402.

12 Sayah DM, Mallavia B, Liu F, et al. Neutrophil extracellular traps are pathogenic in primary graft dysfunction after lung transplantation. Am J Respir Crit Care Med 2015; 191: 455-463.

13 Yildiz C, Palaniyar N, Otulakowski G, et al. Mechanical ventilation induces neutrophil extracellular trap formation. Anesthesiology 2015; 122: 864-875.

14 Avlonitis VS, Fisher AJ, Kirby JA, et al. Pulmonary transplantation: the role of brain death in donor lung injury. Transplantation 2003; 75: 1928-1933.

15 Watts RP, Thom O, Fraser JF. Inflammatory signalling associated with brain dead organ donation: from brain injury to brain stem death and posttransplant ischaemia reperfusion injury. J Transplant 2013; 2013: 521369.

16 Klein SL, Flanagan KL. Sex differences in immune responses. Nat Rev Immunol 2016; 16: 626-638.

17 Simao RR, Ferreira SG, Kudo GK, et al. Sex differences on solid organ histological characteristics after brain death. Acta Cir Bras 2016; 31: 278-285.

18 Hoppenbrouwers T, Autar ASA, Sultan AR, et al. In vitro induction of NETosis: comprehensive live imaging comparison and systematic review. PLoS One 2017; 12: e0176472.

19 Hakkim A, Furnrohr BG, Amann K, et al. Impairment of neutrophil extracellular trap degradation is associated with lupus nephritis. Proc Natl Acad Sci USA 2010; 107: 9813-9818.

20 Mordant $\mathrm{P}$, Nakajima D, Kalaf R, et al. Mesenchymal stem cell treatment is associated with decreased perfusate concentration of interleukin-8 during ex vivo perfusion of donor lungs after 18-hour preservation. J Heart Lung Transplant 2016; 35: 1245-1254.

21 Iskender I, Cosgun T, Arni S, et al. Cytokine filtration modulates pulmonary metabolism and edema formation during ex vivo lung perfusion. J Heart Lung Transplant 2018; 37: 283-291.

22 Machuca TN, Cypel M, Bonato R, et al. Safety and efficacy of ex vivo donor lung adenoviral IL-10 gene therapy in a large animal lung transplant survival model. Hum Gene Ther 2017; 28: 757-765.

23 Giaglis S, Stoikou M, Sur Chowdhury C, et al. Multimodal regulation of NET formation in pregnancy: progesterone antagonizes the Pro-NETotic effect of estrogen and G-CSF. Front Immunol 2016; 7: 565.

24 Harness-Brumley CL, Elliott AC, Rosenbluth DB, et al. Gender differences in outcomes of patients with cystic fibrosis. J Womens Health (Larchmt) 2014; 23: 1012-1020.

25 Heffernan DS, Dossett LA, Lightfoot MA, et al. Gender and acute respiratory distress syndrome in critically injured adults: a prospective study. J Trauma 2011; 71: 878-883. 